\title{
Network and guest dependent thermal stability and thermal expansion in a trigonal host
}

\author{
VISWANADHA G SARASWATULA, MUKHTAR AHMAD BHAT, SUMAN BHATTACHARYA \\ and BINOY K SAHA* \\ Department of Chemistry, Pondicherry University, Puducherry 605 014, India \\ e-mail: binoypu@yahoo.co.in
}

MS received 18 March 2014; revised 11 July 2014; accepted 12 July 2014

\begin{abstract}
Thermal stability and thermal expansion of bromo trimer synthon mediated hexagonal inclusion compounds of 2,4,6-tris(4-bromophenoxy)-1,3,5-triazine (BrPOT) with dichloromethane (DCM), tetrahydrofuran (THF) and hexamethyl benzene (HMB) and also the guest-free form of BrPOT are reported. Each of these three guests produced two concomitant inclusion compounds with BrPOT. The thermal stability of the solvate lattice increases with decreasing cavity size. The channel network of the DCM inclusion compound is stable only for a few seconds at room temperature outside the mother liquor, whereas the cage network of the DCM solvate is stable for months under similar conditions. Thermal expansions of the lattices depend upon the network, guest content as well as the type of guest molecules. The guest-free form exhibits the least thermal expansion in this series of systems.
\end{abstract}

Keywords. Thermal expansion; thermal stability; inclusion compound; halogen bond.

\section{Introduction}

Generally, a material expands along all directions when it is heated. There are a few reports where the materials contract along one, ${ }^{1,2}$ two $^{3-5}$ or even three ${ }^{6-9}$ dimensions on heating. Negative thermal expansion materials are useful in the preparation of composite materials with zero thermal expansion. ${ }^{10,11}$ On the other hand, materials, showing large expansion along one or more directions on temperature change, could be useful in the design of thermo mechanical actuators., ${ }^{42-15}$ Therefore, it is important to understand how different types of materials respond to the temperature variation in their solid-states.

There are several properties that depend upon the crystal packing of the compounds. For example, polymorphs generally show different physical and chemical properties and therefore open up an interesting avenue of comparative studies on relative stabilities, reactivities, etc., ${ }^{16,17}$ of the different forms. Even though, polymorphism is quite common for the single molecular system, ${ }^{18-23}$ it is still relatively rare for the complexes and inclusion compounds. ${ }^{24-28}$

In recent days the halogen bonding has drawn a considerable attention from the crystal engineering community. ${ }^{29-36}$ To study the dependence of thermal

\footnotetext{
*For correspondence
}

expansion on different networks and guest molecules, we have chosen 2,4,6-tris(4-bromophenoxy)-1,3,5-triazines (BrPOT) as a host molecule, because it enclathrates several guest molecules, mainly in two well defined networks - cage and channel structures formed via halogen trimer synthon. ${ }^{37-39}$ It is also possible to crystallize the guest-free form, ${ }^{40}$ and all these systems are highly symmetric.

It is known that the thermal stability of a solvate depends upon the stability of the host network, the nature of the guest molecule, host-guest interactions, etc. ${ }^{41-46}$ Here, we show large differences in the thermal stabilities of two pairs of solvates of BrPOT, formed concomitantly from their respective solutions, depending upon the host networks. We also have compared thermal expansion of different inclusion compounds and the guest-free form.

\section{Experimental}

\subsection{Synthesis}

2,4,6-tris(4-bromophenoxy)-1,3,5-triazine (BrPOT) was synthesized by following the procedure reported in the literature. ${ }^{38}$ The product was characterized by NMR spectroscopy. ${ }^{1} \mathrm{H}$ NMR $\left(\mathrm{CDCl}_{3}, 400 \mathrm{MHz}\right): \delta 7.49$ $(\mathrm{d}, 8.8 \mathrm{~Hz}, 6 \mathrm{H}), 7.01(\mathrm{~d}, 8.8 \mathrm{~Hz}, 6 \mathrm{H}) .{ }^{13} \mathrm{C}$ NMR $\delta 173.0$, 150.6, 132.7, 123.3, 119.6. 


\subsection{Powder $x$-ray diffraction experiments}

The PXRD spectra of guest-free forms of BrPOT solvates were recorded with $\mathrm{X}$-pert Panalytical diffractometer using $\mathrm{Cu} \mathrm{K} \alpha$ radiation $(\lambda=1.54056 \AA)$. The spectra were recorded with $2 \theta$ ranging from $10^{\circ}$ to $50^{\circ}$ for all the three guest-free forms with a step size of $0.02^{\circ}$. The guest-free forms of BrPOT solvates were prepared by grinding the solvate crystals in a mortar, followed by heating the powdered mass at $150-155^{\circ} \mathrm{C}$ under vacuum.

\subsection{Thermal studies}

DSC and TG experiments for the BrPOT-THF-cageI and BrPOT-DCM-cage-II were carried out in TA Instruments. Crystals were taken out from the mother liquor and dried with tissue paper. The samples were placed in crimped but vented aluminum sample pans for DSC and in open alumina pans for TG experiments. 5-7 mg of sample was used for each case. The samples were heated from $25-260^{\circ} \mathrm{C}$ at a rate of $10^{\circ} \mathrm{C} / \mathrm{min}$.

\subsection{Structure determination}

Crystal data were collected on Xcalibur Oxford Diffraction Ltd. with Mo- $\mathrm{K}_{\alpha}$ radiation $(\lambda=0.71073, \AA)$. Crystals were coated with paraffin oil and grease to reduce guest loss. Empirical absorption correction using spherical harmonics, implemented in SCALE3 ABSPACK scaling algorithm were applied. ${ }^{70}$ The phenyl groups in BrPOT-THF-channel structures are disordered over two orientations with 0.5 occupancy on each site. All non-hydrogen atoms were refined anisotropically and the $\mathrm{H}$ atoms bonded to $\mathrm{C}$ were fixed in calculated positions. Structure solution and refinement were carried out using SHELXS-97 and SHELXL-97 (Sheldrick, 1997). ${ }^{71}$ SQUEEZE routine of PLATON was used to treat the residual electron density due to highly disordered guest molecules. CCDC reference numbers
CCDC 992063-992065, 1013423-1013451 and 10134091013414.

\section{Results and Discussion}

\subsection{Crystal structures}

In this work we have studied six host-guest complexes of BrPOT with tetrahydrofuran (THF), dichloromethane (DCM), and hexamethylbenzene (HMB) guests namely, BrPOT-THF-channel, BrPOT.THF-cage-I, BrPOT.DCM - channel, BrPOT·DCM-cage-II, BrPOT. HMB - channel ${ }^{39}$ and BrPOT.HMB - cage-II along with one guest-free form of BrPOT, ${ }^{40}$ BrPOT-gf (figure S1 for ORTEP diagram). The host molecule forms two concomitant inclusion compounds with each of DCM, THF and HMB. The two sets of concomitant solvates were obtained by solvent evaporation method from the solution of BrPOT in the respective solvents and the concomitant inclusion compounds of BrPOT with HMB were obtained from the ethylacetate solution of these two compounds (1:1 ratio). This is the first report of inclusion compounds formed concomitantly in the family of 2,4,6-tris(aryloxy)-1,3,5-triazines. All the three channel structures, BrPOT.DCM-channel, BrPOT.THF-channel and BrPOT.HMB-channel are isostructural. On the other hand, the BrPOT.DCMcage-II and BrPOT.HMB-cage-II are isostructural among the cage types of systems. In all the six inclusion structures, the host molecules are assembled via type-II ${ }^{47-51}$ bromo trimer synthons (table 1) to form hexagonal layers parallel to the crystallographic $a b$ plane. The hexagonal cavity walls are mostly constructed by the phenoxy groups of the host molecules. The nodes of the hexagonal layers are made of triazine rings or bromo trimer synthons and are located at alternate positions of the hexagons. Therefore, the 2D packing arrangements of all the three types of structures are similar but they differ mainly in the stacking

Table 1. Interhalogen interaction geometries of the bromo trimer synthons.

\begin{tabular}{|c|c|c|c|c|}
\hline System/solvate & Temperature & Interaction & $D(\AA)$ & $\theta\left(^{\circ}\right)$ \\
\hline BrPOT·DCM-channel & $118 \mathrm{~K}$ & $\mathrm{Br}_{1} \cdots \mathrm{Br}_{1}$ & $3.463(2)$ & $111.7(3), 171.7(3)$ \\
\hline BrPOT·DCM-cage-II & $118 \mathrm{~K}$ & $\mathrm{Br}_{1} \cdots \mathrm{Br}_{1}$ & $3.625(1)$ & $104.1(2), 162.7(2)$ \\
\hline BrPOT-THF-channel & $120 \mathrm{~K}$ & $\mathrm{Br}_{1} \cdots \mathrm{Br}_{1}$ & $3.475(1)$ & $110.2(1), 170.2(1)$ \\
\hline \multirow{3}{*}{ BrPOT.THF-cage-I } & $120 \mathrm{~K}$ & $\mathrm{Br}_{1} \cdots \mathrm{Br}_{1}$ & $3.493(2)$ & $110.9(3), 170.8(3)$ \\
\hline & & $\mathrm{Br}_{2} \cdots \mathrm{Br}_{2}$ & $3.561(2)$ & $104.9(2), 164.2(2)$ \\
\hline & & $\mathrm{Br}_{3} \cdots \mathrm{Br}_{3}$ & $3.485(2)$ & $112.6(3), 172.6(3)$ \\
\hline BrPOT·HMB-channel & $120 \mathrm{~K}$ & $\mathrm{Br}_{1} \cdots \mathrm{Br}_{1}$ & $3.448(2)$ & $111.4(3), 171.4(3)$ \\
\hline BrPOT·HMB-cage-II & $118 \mathrm{~K}$ & $\mathrm{Br}_{1} \cdots \mathrm{Br}_{1}$ & $3.817(6)$ & $107.4(8), 164.7(9)$ \\
\hline
\end{tabular}



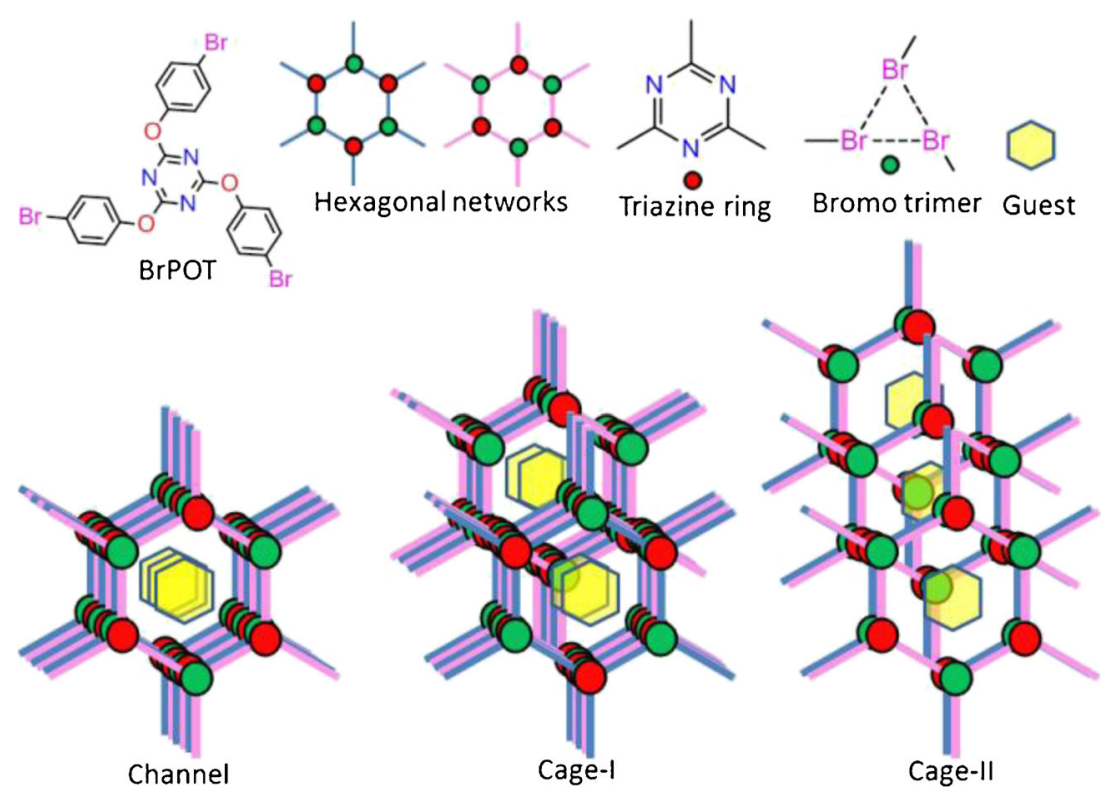

Scheme 1. Schematic representation of the channel, cage-I and cage-II structures made of bromo trimer mediated hexagonal 2D layers of BrPOT host. Consecutive layers are shown in different colours (magenta and blue) for better visualization. The bromo trimer synthons and triazine rings located at the nodes of the hexagonal frameworks are shown in green and red colours respectively. The guest molecules in the cavities are shown in transparent yellow colour.

of the layers in the $3^{\text {rd }}$ dimension (scheme 1). Except in BrPOT.HMB - cage-II structure, the guest molecules in the cavities are highly disordered and could not be modeled from the difference Fourier electron density map and hence SQUEEZE routine of PLATON has been used to refine the structures. ${ }^{52,53}$

The isostructural channel structures of the rod shaped crystals (figure S2) of BrPOT.THF-channel, BrPOT.DCM-channel and BrPOT.HMB-channel, solved in the $P 6_{3} / m$ space group (table 2) with onesixth of the host molecule in the asymmetric unit, are made of hexagonal layers stacked along the $c$ axis without any offset, where two consecutive layers are related by inversion symmetry. According to the SQUEEZE routine, the host:guest ratios in the two solvates structures are 2:1 and 1:1 respectively, whereas according to ${ }^{1} \mathrm{H}-\mathrm{NMR}$ study the host to the guest ratio in BrPOT. HMB-channel is $1: 1$. The triazine ring of the host molecules and the bromo trimer synthons are stacked alternately along the $c$ axis. Therefore, the repeating unit of the nodes along the $c$ axis is an infinite array of -triazine-bromo trimer synthon-triazine-bromo trimer synthon- type (figure 1a). The honeycomb channel walls are constructed by the infinite layers of phenoxy groups of the host molecules.

The diffraction quality single crystal of the cage I structure of BrPOT-THF-cage-I inclusion compound, obtained concomitantly with the BrPOT.THF-channel crystals, is solved in $P 6_{3} / m$ space group (table 2) with two of the one-third and one of the one-sixth of host molecules in the symmetry independent unit. These three molecules form three independent halogen trimer synthons (table 1). From the ${ }^{1} \mathrm{H}-\mathrm{NMR}$ and thermal gravimetric (TG) experiments, the host to the guest ratio is found to be 1:2 (figure 2 and table S1). The hexagonal 2D layers are stacked like the channel structure but with some offset. A set of consecutive five layers remains intact, similar to the channel structure, and the next adjacent set of five layers are shifted by a distance equal to the radius of the hexagon to construct the Cage-I structure (figure 1b). This network, formed by a 2,4,6-tris(4-halophenoxy)-1,3,5-triazine inclusion compound, has not been reported previously. Along the $c$ axis, the repeating unit of the nodes is inversion related ten layers of triazine-bromo trimer synthon-triazinebromo trimer synthon-triazine-triazine-bromo trimer synthon-triazine-bromo trimer synthon-triazine and the two stacked triazine rings form a $C_{3 i}$-Piedfort Unit around the inversion center. ${ }^{54-56}$ The cavities are located along the $(1 / 3,1 / 3, c)$ and $(2 / 3,2 / 3, c)$ axes in the unit cell and each cavity is capped by inversion related five layers of bromo trimer synthon-triazine-bromo trimer synthon-triazine-bromo trimer synthon units from both the sides along these axes. As a result the side wall of the cavity in this structure is made of five layers of phenoxy groups. 
The isostructural block shaped crystals of the BrPOT.DCM-cage-II and BrPOT.HMB-cage-II inclusion compounds, which formed concomitantly along with the respective rod shaped (BrPOT.DCM-channel and BrPOT.HMB - channel respectively) crystals, are solved in $R \overline{3}$ space group (table 2 ) with one third of the host molecule in the asymmetric units. Though the highly disordered DCM guest molecules could not be modeled in the BrPOT.DCM-cage-II structure, the ordered one-sixth of the HMB guest molecule has been located in the asymmetric unit of the BrPOT. $\mathrm{HMB}$-cage-II structure. The ${ }^{1} \mathrm{H}-\mathrm{NMR}$ and TG experiments suggest a 1:1 host to the guest ratio in the BrPOT.DCM-cage-II inclusion complex. In the cageII structure, the 2D layers are stacked with some offset but is distinct from the cage-I network and generate a different type of cage like structure, cage-II (figure 1c). With respect to the bottom two layers, there is a

Table 2. Crystallographic table.

\begin{tabular}{|c|c|c|c|c|}
\hline System & $\begin{array}{l}\text { BrPOT-gf, } \\
118 \mathrm{~K}\end{array}$ & $\begin{array}{l}\text { BrPOT.DCM- } \\
\text { channel, } 118 \mathrm{~K}\end{array}$ & $\begin{array}{c}\text { BrPOT.THF- } \\
\text { channel, } 120 \mathrm{~K}\end{array}$ & $\begin{array}{l}\text { BrPOT.HMB- } \\
\text { channel, } 120 \mathrm{~K}\end{array}$ \\
\hline Formula & $\mathrm{C}_{21} \mathrm{H}_{12} \mathrm{Br}_{3} \mathrm{~N}_{3} \mathrm{O}_{3}$ & $\mathrm{C}_{22} \mathrm{H}_{14} \mathrm{Br}_{3} \mathrm{Cl}_{2} \mathrm{~N}_{3} \mathrm{O}_{3}$ & $\mathrm{C}_{23} \mathrm{H}_{16} \mathrm{Br}_{3} \mathrm{~N}_{3} \mathrm{O}_{3.5}$ & $\mathrm{C}_{27} \mathrm{H}_{21} \mathrm{Br}_{3} \mathrm{~N}_{3} \mathrm{O}_{3}$ \\
\hline$M \mathrm{r}$ & 594.07 & 678.99 & 630.12 & 675.20 \\
\hline Crystal system & trigonal & hexagonal & hexagonal & hexagonal \\
\hline Space group & $R 3 \mathrm{c}$ & $\mathrm{Pb}_{3} / \mathrm{m}$ & $P 6_{3} / \mathrm{m}$ & $P 6_{3} / \mathrm{m}$ \\
\hline$a(\AA)$ & $23.9189(8)$ & $15.6381(16)$ & $15.6462(7)$ & $15.615(4)$ \\
\hline$b(\AA)$ & $23.9189(8)$ & $15.6381(16)$ & $15.6462(7)$ & $15.615(4)$ \\
\hline$c(\AA)$ & $6.4691(3)$ & $6.9069(5)$ & $6.8645(3)$ & $6.8639(17)$ \\
\hline$\alpha\left(^{\circ}\right)$ & 90.00 & 90.00 & 90.00 & 90.00 \\
\hline$\beta\left(^{\circ}\right)$ & 90.00 & 90.00 & 90.00 & 90.00 \\
\hline$\gamma\left(0^{\circ}\right)$ & 120.00 & 120.00 & 120.00 & 120.00 \\
\hline$V\left(\AA^{3}\right)$ & $3205.2(2)$ & $1462.8(2)$ & $1455.32(12)$ & $1449.3(6)$ \\
\hline Crystal size (mm) & $0.36 * 0.16 * 0.14$ & $0.4 * 0.25 * 0.2$ & $0.6 * 0.5 * 0.4$ & $0.5 * 0.4 * 0.3$ \\
\hline$T(\mathrm{~K})$ & $118(2)$ & $118(2)$ & $120(2)$ & $120(2)$ \\
\hline $\mathrm{Z}$ & 6 & 2 & 2 & 2 \\
\hline$F(000)$ & 1728 & 660 & 616 & 666 \\
\hline$\mu\left(\mathrm{mm}^{-1}\right)$ & 5.692 & 4.344 & 4.184 & 4.206 \\
\hline Ref. collected/unique & 1543 & 1297 & 1275 & 1233 \\
\hline Parameters & 91 & 55 & 73 & 55 \\
\hline Final $\mathrm{R}$ indices $[\mathrm{I}>2 \sigma(\mathrm{I})]$ & 0.0313 & 0.0734 & 0.0352 & 0.0718 \\
\hline $\mathrm{R}$ indices (all data) & 0.0708 & 0.2088 & 0.0944 & 0.1673 \\
\hline Goodness of fit on $F^{2}$ & 1.037 & 1.012 & 1.138 & 1.081 \\
\hline System & $\begin{array}{l}\text { BrPOT.DCM- } \\
\text { cage-II, } 118 \text { K }\end{array}$ & $\begin{array}{l}\text { BrPOT.THF- } \\
\text { cage-I, } 120 \mathrm{~K}\end{array}$ & $\begin{array}{l}\text { BrPOT.HMB- } \\
\text { cage-II, 118K }\end{array}$ & \\
\hline Formula & $\mathrm{C}_{22} \mathrm{H}_{14} \mathrm{Br}_{3} \mathrm{Cl}_{2} \mathrm{~N}_{3} \mathrm{O}_{3}$ & $\mathrm{C}_{29} \mathrm{H}_{28} \mathrm{Br}_{3} \mathrm{~N}_{3} \mathrm{O}_{5}$ & $\mathrm{C}_{54} \mathrm{H}_{42} \mathrm{Br}_{6} \mathrm{~N}_{6} \mathrm{O}_{6}$ & \\
\hline$M \mathrm{r}$ & 678.99 & 738.27 & 1350.40 & \\
\hline Crystal system & trigonal & hexagonal & trigonal & \\
\hline Space group & $R \overline{3}$ & $P 6_{3} / \mathrm{m}$ & $R \overline{3}$ & \\
\hline$a(\AA)$ & $15.0828(10)$ & $15.5333(9)$ & $15.3892(5)$ & \\
\hline$b(\AA)$ & $15.0828(10)$ & $15.5333(9)$ & $15.3892(5)$ & \\
\hline$c(\AA)$ & $18.5405(14)$ & $33.553(2)$ & $18.1170(8)$ & \\
\hline$\alpha\left(^{\circ}\right)$ & 90.00 & 90.00 & 90.00 & \\
\hline$\beta\left(^{\circ}\right)$ & 90.00 & 90.00 & 90.00 & \\
\hline$\gamma\left({ }^{\circ}\right)$ & 120.00 & 120.00 & 120.00 & \\
\hline$V\left(\AA^{3}\right)$ & $3652.7(4)$ & $7011.3(7)$ & $3715.7(2)$ & \\
\hline Crystal size $(\mathrm{mm})$ & $0.45 * 0.4 * 0.38$ & $0.48 * 0.36 * 0.32$ & $0.55 * 0.4 * 0.2$ & \\
\hline$T(\mathrm{~K})$ & $118(2)$ & $120(2)$ & $118(2)$ & \\
\hline $\mathrm{Z}$ & 6 & 10 & 3 & \\
\hline$F(000)$ & 1980 & 3680 & 1998 & \\
\hline$\mu\left(\mathrm{mm}^{-1}\right)$ & 5.219 & 4.360 & 4.922 & \\
\hline Ref. collected/unique & 1349 & 5570 & $4624 / 1899$ & \\
\hline Parameters & 91 & 235 & 110 & \\
\hline Final $\mathrm{R}$ indices $[\mathrm{I}>2 \sigma(\mathrm{I})]$ & 0.0555 & 0.0863 & 0.0304 & \\
\hline $\mathrm{R}$ indices (all data) & 0.1700 & 0.2259 & 0.0687 & \\
\hline Goodness of fit on $F^{2}$ & 1.113 & 1.047 & 1.031 & \\
\hline
\end{tabular}




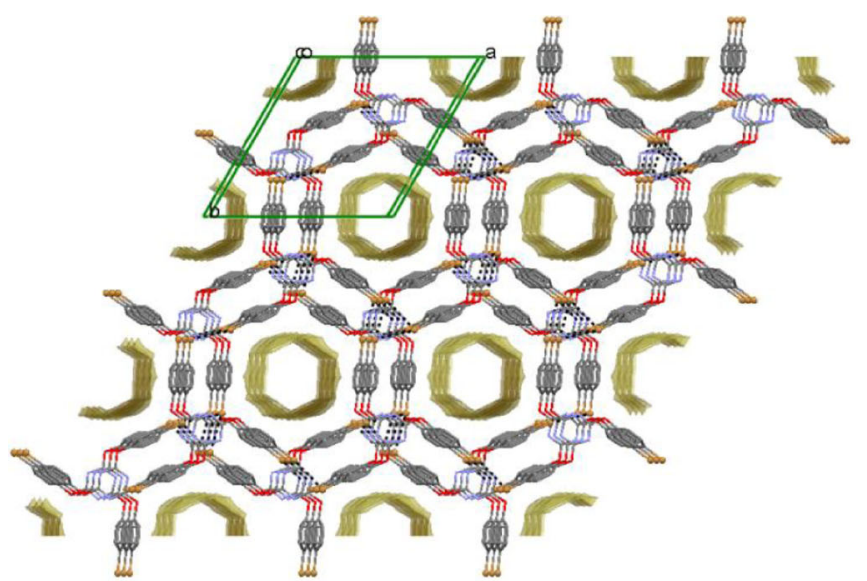

(a)

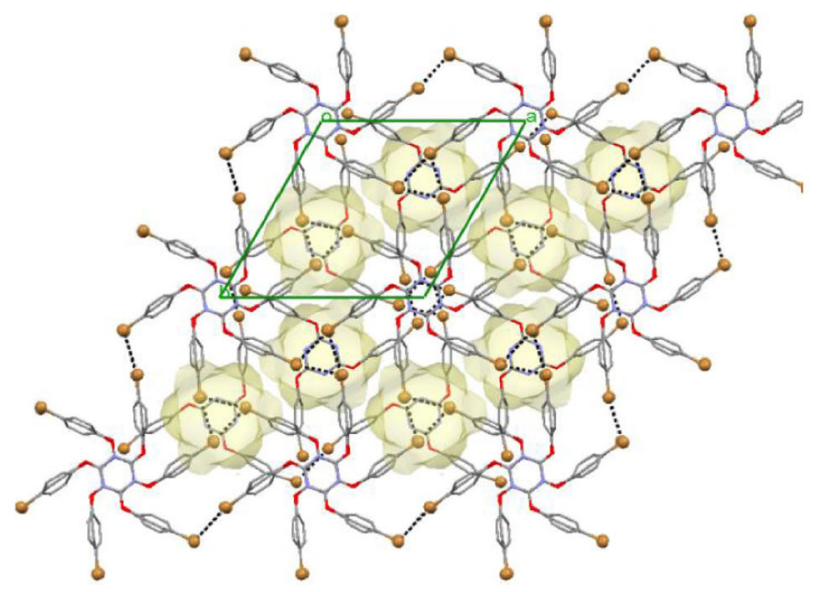

(b)

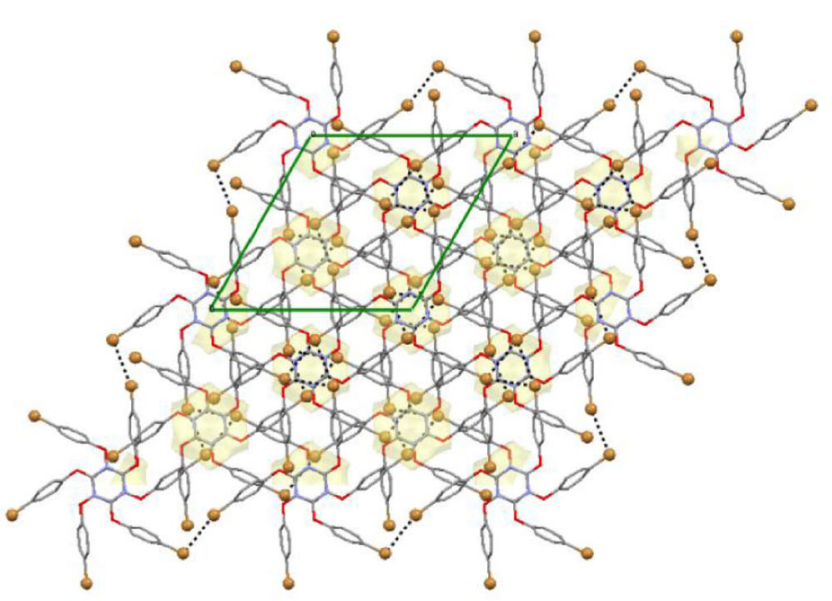

(c)

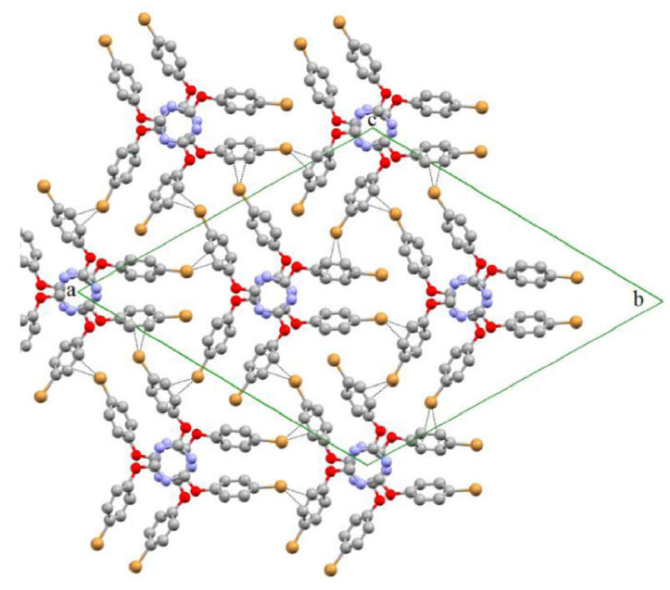

(d)

Figure 1. The bromo trimer synthon mediated 2D honeycomb layers are stacked along the $c$ axis with different offsets to form (a) BrPOT.DCM-channel, (b) cage-I, and (c) cage-II structures. The cavities are represented by yellow coloured space and the bromine atoms are shown as spheres. (d) Packing diagram of the BrPOT molecules in the guest-free form.

translation of the next pair of layers by a distance equal to the radius of the hexagon and the $5^{\text {th }}$ and $6^{\text {th }}$ layers also move by the same distance with respect to the $3^{\text {rd }}$ and $4^{\text {th }}$ layers in the same direction to complete the repeating unit along the $c$ axis. Due to this off set stacking of the layers, voids are created in the hexagonal network. The side-walls of each of these hexagonal voids are constructed by the two layers of phenoxy groups of the host molecules and the guest molecules in the cavity are embedded between an inversion related pair of bromo trimer synthon and triazine ring to form a pentadecker sandwich of triazine-bromo trimer synthon-cavity-bromo trimer synthon-triazine, which is the repeating unit along the $c$ axis. In this type of structure the triazine rings are stacked in a $C_{3 i}$-PU similar to the cage-I structure.

The guest-free form crystallizes in $R 3 c$ space group with only $1 / 3$ molecule of the compound in the asymmetric unit (table 2). In contrary to the host networks, the crystal structure is devoid of any $\mathrm{Br} \cdots \mathrm{Br}$ interactions. Rather, the bromo groups are involved in $\mathrm{Br} \cdots \pi$ interactions in this crystal structure. The molecules are stacked atop each other and form molecular rod and these rods are arranged in a hexagonal array via $\mathrm{Br} \cdots \pi$ interactions (figure $1 \mathrm{~d}$ ).

\subsection{Thermal stability}

This series of structures show an interesting structurethermal stability relationship. The cage types of structures are thermally more stable than the channel type of networks. The 2D arrangements of the host molecules in these structures are basically similar and they differ mainly in the $3^{\text {rd }}$ dimension. As a result, all the three types of cavities, generated in these host networks, are hexagonal and are of similar diameter, but 
the heights of the cavities change and hence the sizes of the cavities also differ. The cavity size in the channel is largest which is essentially infinite along the channel axis, whereas it is smallest in the case of cageII structure. Interestingly, the channel and the cageI or II clathrates of BrPOT form concomitantly from the THF or DCM solution of the host respectively. Therefore, these pseudo polymorphs are also practically of similar energy like concomitant polymorphs. In general, the physical properties of the polymorphs do not differ widely, ${ }^{57-61}$ but in the case of two DCM solvates the thermal stabilities of the systems differ quite dramatically. The rod shaped single crystals (BrPOT·DCM-channel) were turned opaque within a few seconds after they were taken out from the mother liquor, whereas the distinct block shaped crystals (BrPOT·DCM-cage II) were stable for months at room temperature outside the mother liquor. On the other hand, the crystals of the cage-I THF clathrate were stable for a few minutes, and the concomitantly formed channel type THF clathrate crystals were stable only for 1-2 minutes at ambient condition outside the mother liquor. Even though, the boiling point of THF $\left(66^{\circ} \mathrm{C}\right)$ is considerably higher than that of DCM $\left(39.6^{\circ} \mathrm{C}\right)$ guest, the cage-II type DCM clathrate is thermally the most stable system among these four solvate systems. Thermal analysis shows that the onset temperature of the DCM molecules in the cage-II network is around $65^{\circ} \mathrm{C}$, whereas in the case of THF molecules for the cage-I framework it is only $45^{\circ} \mathrm{C}$ (figure 2). There is also an interesting correlation between the thermal stability and cavity size in this series of networks. PLATON calculation shows that the $\%$ of solvent accessible volume

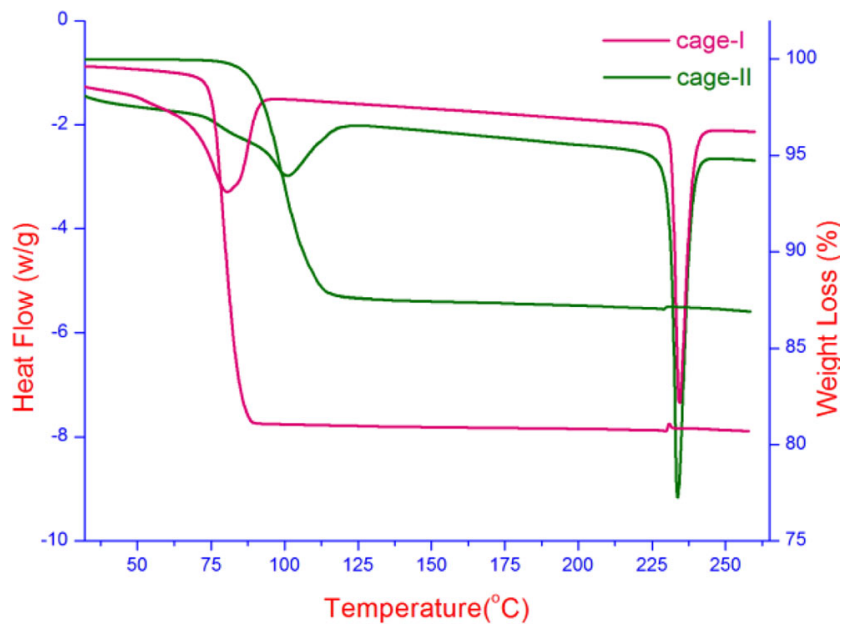

Figure 2. DSC and TG plots of BrPOT.THF-cage-I (purple) and BrPOT.DCM-cage-II (green) structures of BrPOT. The guest release in the case of cage-II occurs at higher temperature than cage-I.

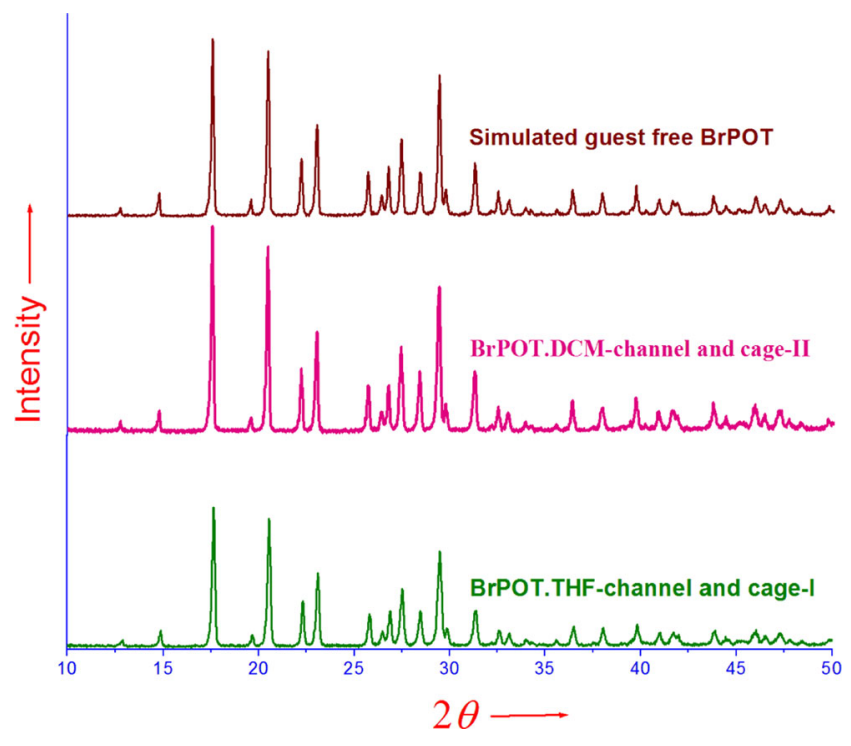

Figure 3. PXRD pattern of the solids after removal of the guests from BrPOT-THF-channel and cage-I, BrPOT·DCMchannel and cage-II, and the simulated guest-free form of BrPOT.

and the size of each cavity decreases as channel (36\%, infinite) >cage-I $\left(33 \%, 1158 \AA^{3}\right)>$ cage-II $(23 \%$, $280 \AA^{3}$ ). Therefore, the cage-II structure is host like, whereas in the channel structure the guest properties dominate compared to the other structures and hence more susceptible to the thermal vibrations. As a result, the thermal stability of the systems increases as the size of the cavity decreases. Interestingly, after removal of guest molecules from the lattices, all the three types of networks collapse to the same guest free form of BrPOT as seen by PXRD analysis (figure 3).

\subsection{Thermal expansion study}

We have compared thermal expansion properties of these seven systems by analyzing single crystal X-ray diffraction data at 5 or 6 different temperatures (table S2). According to the calculation using PASCal ${ }^{62}$ program, all the systems show positive thermal expansion along all the three principal axes (figure 4) and the major axis is aligned along the crystallographic $c$ axis in all these cases (table S3). Interestingly, thermal expansion along the unique axis $c$, i.e., perpendicular to the central triazine plane, is 2.5-5 times higher than the expansion along the other two principal axes, which are aligned in the plane of bromo trimer mediated 2D sheets. Along the $c$ axis, the interactions are mainly of weak $\pi \cdots \pi$ types, whereas in the $2 \mathrm{D}$ plane the molecules are assembled via $\mathrm{Br} \cdots \mathrm{Br}$ (in the inclusion compounds) or $\mathrm{Br} \cdots \pi$ (in the guest-free form) 


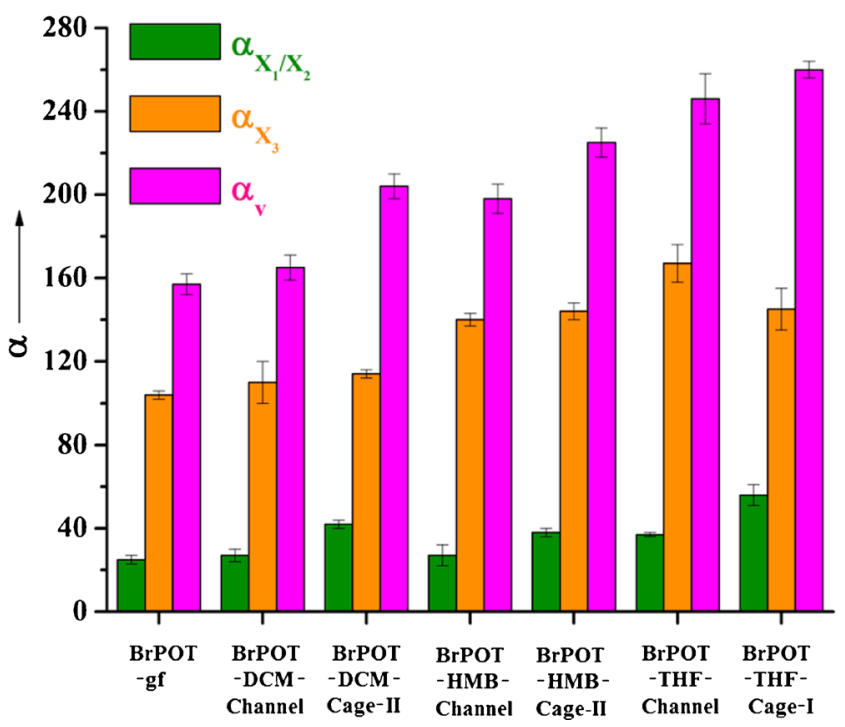

Figure 4. Thermal expansion coefficients along the major axis $\left(\alpha_{\mathrm{x} 3}\right)$, medium/minor axes $\left(\alpha_{\mathrm{x} 2 / 1}\right)$ and in volume $\left(\alpha_{\mathrm{v}}\right)$ are plotted for the seven systems.

interaction (table $\mathrm{S} 4) . \mathrm{C}-\mathrm{H} \cdots \mathrm{N}$ and $\mathrm{C}-\mathrm{H} \cdots \mathrm{O}$ interactions contribute along both the directions (table S5). The near perpendicular conformation of the phenoxy groups with respect to the central triazine ring also might play some role in this anisotropic thermal expansion. The volumetric thermal expansion in the guestfree form is lowest $\left(\alpha_{\mathrm{v}}=157(5) \mathrm{MK}^{-1}\right)$ among these seven systems. The channel structures show comparatively lower thermal expansion than the corresponding cage structures and in fact thermal expansion in BrPOT.DCM-channel $\left(\alpha_{\mathrm{v}}=165(6) \mathrm{MK}^{-1}\right)$ is comparable to the guest-free form. Previously we had shown that thermal expansions of solvates are $\sim 25 \%$ more than that of guest-free forms in a series of interhalogen interaction mediated organic lattices. ${ }^{63}$ Relatively small thermal expansion in the channel structures, especially in the case of BrPOT.DCM-channel system, could be due to lower guest content of the channels. In spite of having solid guest molecule in the cavity, the corresponding isostructural channel structure of BrPOT. HMB-channel $\left(\alpha_{\mathrm{v}}=198(7) \mathrm{MK}^{-1}\right)$ exhibits stronger thermal expansion than that of BrPOT.DCM-channel but smaller thermal expansion than the BrPOT. HMB-cage-II $\left(\alpha_{\mathrm{v}}=225(7) \mathrm{MK}^{-1}\right)$ structure. Due to very unstable nature of the lattices of the channel type of solvates, some of the guest molecules might have escaped during sample handling and experiments. Detail quantitative study could not be performed because of thermal instability of the lattices even at ambient condition. Except very few examples, generally the host networks of organic solvates do not sustain after the guest loss. ${ }^{32,64-66}$ Previously, lower thermal expansion due to low guest content have been shown only in the case of metal-organic frameworks. Thermal vibration of the guest molecules along the open channel axis does not cause expansion in the host network. This could be another reason for lower thermal expansion in the channel structures.

\section{Conclusion}

In this work we have studied three different types of host networks of the BrPOT host. Three pairs of the inclusion compounds of BrPOT formed concomitantly with the DCM, THF and HMB guests. These three types of structures are 2D isostructural ${ }^{67-69}$ but differ in the cavity size due to differences in the $3^{\text {rd }}$ dimension. The concomitant pseudo polymorphs of the inclusion compounds are widely different in their thermal stability. The cage types of structures are more stable than the channel structures and also the structure with the larger cavity size is thermally less stable irrespective of the boiling point of the liquid guest molecules. The channel structures have opening and the solvent guest molecules could escape easily through these openings, whereas in the cage structures the guest molecules have to rupture the host network to escape from the lattice. Thermal expansions of the solvates are higher than the guest-free forms. Thermal expansion among the inclusion compounds also depends upon the network as well as type of guest molecules and the content of guest molecule incorporated in the cavities.

\section{Supplementary Information}

ORTEP plots, pictures of the single crystals, crystallographic information table, thermal expansion coefficient values, and interhalogen interaction geometry are given. Electronic supplementary information can be seen at www.ias.ac.in/chemsci.

\section{Acknowledgements}

B K S thanks CSIR for research funding (No. 02(0026)/11/EMR-II, dated 16/12/11). We gratefully thank DST, India for SXRD facility, Dr. R N Bhowmik, Dept. of Physics, Pondicherry University for PXRD facilities, C.I.F, P.U. for TG-DSC and NMR facilities. V G S thanks UGC, M.A.B thanks CSIR and S.B. thanks Pondicherry University for fellowships.

\section{References}

1. Bhattacharya S and Saha B K 2012 Cryst. Growth Des. 124716 
2. Bhattacharya S and Saha B K 2014 CrystEngComm. 16 2340

3. Das D, Jacobs T and Barbour L J 2010 Nat. Mater. 936

4. Grobler I, Smith V J, Bhatt P M, Herbert S A and Barbour L J 2013 J. Am. Chem. Soc. 1356411

5. Bhattacharya S, Saraswatula V G and Saha B K 2013 Cryst. Growth Des. 133651

6. Grima J N, Zammit V and Gatt R 2006 Xjenza 1117

7. Lind C 2012 Materials 51125

8. Salvador J R, Guo F, Hogan T and Kanatzidis M G 2003 Nature 425702

9. Hu P, Chen J, Sun X, Deng J, Chen X, Yu R, Qiao L and Xing X J 2009 Mater. Chem. 191648

10. Miller K J, Romao C P, Bieringer M, Marinkovic B A, Prisco L and White M A 2013 J. Am. Ceram. Soc. 96 561

11. Zhang X, Ren Y, Roushan M and Li J 2012 Eur. J. Inorg. Chem. 5966

12. Li L, Xu X, Chew H, Huang X, Dou X, Pan S, Li G and Zhang L 2008 J. Phys. Chem. C 1125328

13. Noailles L D, Peng H -H, Starkovich J and Dunn B 2004 Chem. Mater. 161252

14. Dubbeldam D, Walton K S, Ellis D E and Snurr R Q 2007 Angew. Chem., Int. Ed. 464496

15. DeVries L D, Barron P M, Hurley E P, Hu C and Choe W 2011 J. Am. Chem. Soc. 13314848

16. Chen S, Xi H and Yu L 2005 J. Am. Chem. Soc. 127 17439

17. Chen X, Morris K R, Griesser U J, Byrn S R and Stowell J G 2002 J. Am. Chem. Soc. 12415012

18. Cruz-Cabeza A J and Bernstein J 2014 Chem. Rev. 114 2170

19. Tothadi S, Bhogala B R, Gorantla A R, Thakur T S, Jetti R K R and Desiraju G R 2012 Chem. Asian J. 7330

20. Kumar S S and Nangia A 2013 CrystEngComm 156498

21. Dikundwar A G, Dutta G K, Row T N G and Patil S 2011 Cryst. Growth Des. 111615

22. Roy S, Banerjee R, Nangia A and Kruger G J 2006 Chem. Eur. J. 123777

23. Krishna G R, Kiran M S R N, Fraser C L, Ramamurty U and Reddy C M 2013 Adv. Funct. Mater. 231422

24. Lalit Rajput and Kumar Biradha 2011 J. Mol. Struct. 991 97

25. Suzuki M and Kobayashi K 2011 Cryst. Growth Des. 11 1814

26. Zhang Q, Lu L, Dai W and Mei X 2014 CrystEngComm 161919

27. Bhattacharya S, Stojakovi J, Saha B K and MacGillivray L R 2013 Org let. 15744

28. Bhattacharya S and Saha B K 2011 Cryst. Growth Des. 112194

29. Abate A, Martí-Rujas J, Metrangolo P, Pilati T, Resnati G and Terraneo G 2011 Cryst. Growth Des. 114220

30. Caronna T, Liantonio R, Logothetis T A, Metrangolo P, Pilati T and Resnati G 2004 J. Am. Chem. Soc. 1264500

31. Choudhury A R and Row T N G 2004 Cryst. Growth Des. 447

32. Bhattacharya S and Saha B K 2012 Cryst. Growth Des. 12169

33. Forni A, Metrangolo P, Pilati T and Resnati G 2004 Cryst. Growth Des. 4291

34. Metrangolo P, Murray J S, Pilati T, Politzer P, Resnati G and Terraneo G 2011 Cryst. Growth Des. 11 4238
35. Hathwar V R, Gonnade R G, Munshi P, Bhadbhade M M and Row T N G 2011 Cryst. Growth Des. 11 1855

36. Moorthy J N, Venkatakrishnan P, Mal P, Dixit S and Venugopalan P 2003 Cryst. Growth Des. 3581

37. Saha B K, Jetti R K R, Reddy L S, Aitipamula S and Nangia A 2005 Cryst. Growth Des. 5887

38. Jetti R K R, Xue F, Mak T C W and Nangia A 1999 Cryst. Eng. 2215

39. Anthony A, Desiraju G R, Jetti R K R, Kuduva S S, Madhavi N N L, Nangia A, Thaimattam R and Thalladi V R 1998 Cryst. Eng. 11

40. Reichenbacher K, Suss H I, Stoeckli-Evans H, Bracco S, Sozzani P, Weber E and Hulliger J 2004 New J. Chem. 28393

41. Nassimbeni L R 2003 Acc. Chem. Res. 36631

42. Nassimbeni L R and Su H 2002 J. Chem. Soc., Perkin Trans. 21246

43. Nassimbeni, L R 2004 In Encyclopedia of supramolecular chemistry J L Atwood and J W Steed (Eds.) (U.S.A.: Marcel Dekker) pp 696-704

44. Nassimbeni L R 2003 CrystEngComm 5200

45. Skobridis K, Theodorou V, Seichter W and Weber E 2010 Cryst. Growth Des. 10862

46. Das D and Barbour L J 2008 Chem. Commun. 5110

47. Desiraju G R and Parthasarathy R J 1989 J. Am. Chem. Soc. 1118725

48. Dey A, Jetti R K R, Boese R and Desiraju G R 2003 CrystEngComm $\mathbf{5} 248$

49. Pigge F C, Vangala V R and Swenson D C 2006 Chem. Commun. 2123

50. Bosch E and Barnes C L 2002 Cryst. Growth Des. 2 299

51. Saha B K and Nangia A 2007 Heteroatom Chem. 18 185

52. Spek A L 2003 J. Appl. Cryst. 367

53. Spek A L 2009 Acta Crystallogr. Sect. D 65148

54. Jessiman A S, MacNicol D D, Mallinson P R and Vallance I 1990Chem. Commun. 1619

55. Thalladi V R, Brasselet S, Weiss H C, Bläser D, Katz A K, Carrell H L, Boese R, Zyss J, Nangia A and Desiraju G R 1998 J. Am. Chem. Soc. 1202563

56. Saha B K, Aitipamula S, Banerjee R, Nangia A, Jetti R K R, Boese R, Lam C-K and Mak T C W 2005 Mol. Cryst. Liq. Cryst. 440295

57. Babu N J, Cherukuvada S, Thakuria R and Nangia A 2010 Cryst. Growth Des. 101979

58. Munshi P, Venugopala K N, Jayashree B S and Row T N G 2004 Cryst. Growth Des. 41105

59. Munshi P and Row T N G 2006 Cryst. Growth Des. 6 708

60. Muguruma H and Hotta S 2006 J. Phys. Chem. B 110 23075

61. Erk P, Hengelsberg H, Haddowb M F and Gelder R V 2004 CrystEngComm 6474

62. Cliffe M J and Goodwin A L 2012 J. Appl. Cryst. 45 1321

63. Saraswatula V G and Saha B K 2014 New J. Chem. 38 897

64. Saha B K and Nangia A 2006 CrystEngComm 8440

65. Saha B K and Nangia A 2005 Chem. Commun. 3024

66. Bhattacharya S and Saha B K 2013 Cryst. Growth Des. 13606 
67. Das D and Barbour L J 2009 Crystal Growth Des. 9 1599

68. Bhattacharya S, Sameena J and Saha B K 2011 Cryst. Growth Des. 11905

69. Banerjee R, Bhatt P M and Desiraju G R 2006 Crystal Growth Des. 61468
70. CrysAlisPro, Oxford Diffraction Ltd., Version 1.171.33.44, 2010

71. Sheldrick G M, SHELXS-97 and SHELXL-97, Programs for the Solution and Refinement of Crystal Structures, University of Göttingen, Germany, 1997 